\title{
Familial Isolated Hyperparathyroidism
}

National Cancer Institute

\section{Source}

National Cancer Institute. Familial Isolated Hyperparathyroidism. NCI Thesaurus. Code C94830.

A rare, autosomal dominant hereditary syndrome characterized by hypercalcemia, abnormally high levels of parathyroid hormone, and isolated hyperfunctioning parathyroid tumors. 